\title{
MicroED: Tiny Crystals, Big Opportunities
}

Michael W. Martynowycz ${ }^{\mathrm{a}}$, Johan Hattne ${ }^{\mathrm{a}}$, and Tamir Gonen ${ }^{\mathrm{a}}$

aHoward Hughes Medical Institute, Departments of Biological Chemistry and Physiology, University of California Los Angeles, Los Angeles CA, 90095, USA, mikewm@g.ucla.edu

In drug discovery, unambiguous identification of small molecules is of paramount importance. Micro-crystal electron diffraction (MicroED) is an electron cryo-microscopy (cryoEM) method that determines atomic structures from nanocrystals. We start from apparently amorphous powders, and demonstrate atomic resolution $(<1 \AA)$ structures just minutes after loading them onto a modern transmission electron microscope (TEM). This presentation introduces MicroED as a tool for small-molecule structure determination. Results from recent studies are presented along with current developments in drug discovery, protein-ligand interactions, and advances sample preparation techniques. 